\title{
Overview of Metabiotics and Probiotic Cultures During Fermentation of Molasses
}

\author{
V.S. Popov*1, N.V. Vorobyeva1', G.A. Svazlyan¹, N.M. Naumov¹, O.A. Gryaznova²
}

${ }^{1}$ Federal State Budgetary Scientific Institution “Kursk Federal Agricultural Research Center” Kursk, Russia

${ }^{2}$ Federal state budgetary educational institution of higher education "Kursk state agricultural Academy named after I. I. Ivanov" Kursk, Russia

Corresponding Author: V. S. Popov, Federal State Budgetary Scientific Institution "Kursk Federal Agricultural Research Center" Kursk, Russia

\begin{abstract}
The development of biological products synthesizing metabiotics - amino acids, vitamins and other biologically active substances in the gastrointestinal tract of animals is considered as the most promising approach to the formation of microbiocenosis of intestinal microflora. The use of metabolic products of probiotic microorganisms (metabiotics) is an actual direction in the study of the metabolism of probiotic microorganisms and a reasonable alternative to the use of feed antibiotics in animal diets. Moreover, the creation of biologically active feed additives based on them is relevant for the correction of intestinal microbiocenosis and stimulation of non-specific immunity. The aim of the research is the scientific and practical justification of fermentation of molasses by probiotic microorganisms and the possibility of creating a biologically active feed additive. The research objectives included the study of the efficiency of cultivation of Clostridium thermocellulociticus, Ruminococcus olbus, Clostridium lochheadii on a nutrient medium from beet molasses; obtaining culture fluid with metabiotics of probiotic microorganisms; explanation of research results to create a dietary supplement for animals. The studies found that during cultivation, the maximum number of probiotic microorganisms that make up the enzymatic probiotic Cellobacterin is $9,9 \times 10^{6} \mathrm{CFU} / \mathrm{cm}^{3}$ in $15 \%$ nutrient medium. According to the test results, the variability of the mass fraction of the amount of fermentable sugars in the range of $53.70 \%-$ $33.79 \%$, the hydrogen index from 6.70 to 3.87 in the experimental samples of cultivated microorganisms was established. A significant excess of Arginin was found to be 2.8 times, Tyrosine 57.7-66.1 times, Leucine + isoleucine 3.90-6.65 times, and other non-replaceable and conditionally replaceable amino acids. The metabiotics of cultivated probiotic agents from the vitamin group are represented mainly by group "B". The content of oxalic acid in the test samples is slightly lower with respect to the control within $3.8 \%-33.2 \%$. The content of acetic acid is lower than the control by $27.4 \%$. The increase in amber acid in all experimental samples is $49-41$ times higher in relation to the control. Thus, the composition of metabiotics provides the basis for further research in the development of biologically active additives for animals.
\end{abstract}

Keywords: Probiotics, metabiotics, metabolites, colony forming units, amino acids, vitamins.

Correspondence:

V.S. Popov

Federal State Budgetary Scientific Institution "Kursk Federal Agricultural Research Center" Kursk, Russia

\section{INTRODUCTION}

The transition to a highly productive and environmentally friendly fauming involves the development and implementation of systems for the rational use of biological protection products for farm animals, and the creation of safe and high-quality, biologically active feed additives. It should be noted that the rejection of the use of feed antibiotics in animal feeding in the EU and the Russian Federation requires an alternative search for ways to increase nonspecific immunity and natural resistance [1-3]. The most promising and relevant direction in this field of research is the study and use of probiotic microorganisms. Probiotics are living microbial supplements that have a beneficial effect on the animal's body by improving the intestinal microbial balance, stimulate metabolic and immune processes. Probiotics are related to correctors of animal productivity, energotrophics (means directing the energy of nutrients to increase animal productivity) [4-6]. These drugs are not vital for the body but are able to increase and stabilize the productivity of animals [7, 8]. The development of biological products (probiotics) synthesizing metabiotics - amino acids, vitamins and other biologically active substances in the gastrointestinal tract of animals, which are carried out in Russia and abroad, are considered as the most promising approach to the formation of microbiocenosis of intestinal microflora. Moreover, Clostridium thermocellulociticus, Ruminococcus olbus, Clostridium lochheadii were studied as producers [9-11]. These microorganisms belong to the "normal" non-pathogenic microflora, the active population of which in the gastrointestinal tract helps to suppress the growth of conditionally pathogenic and pathogenic microflora of animal resistance. The use of probiotic agents in the form of biologically active feed additives is of the highest prority [12-14].

A group of metabiotics containing metabolic products or structural components of probiotic microorganisms has been highlighted in recent years. Their advantages are that metabiotics have high bioavailability, as metabiotic substances reach the colon 95-97\% unchanged (in probiotics it is less than $0.0001 \%$ ); do not conflict (antagonistic relationships) with the body's own microbiota, unlike probiotic microbes; exhibit activity directly in the gastrointestinal tract [15-17]. The preventive effect of metabiotics is due to a combination of several basic actions: the ability to provide the necessary conditions for normal interaction of the epithelium and microflora with homeostasis in the contact zone of the intestine, as well as a direct effect on the physiological functions and biochemical reactions of the macro- 
organism, and on the activity of cells. The body's own microflora is also stimulated [18-20].

Currently, there is an insufficient number of publications in the scientific literature on the study and biotechnological production of various types of biologically active substances produced mainly by sporeforming microorganisms. The main attention of researchers is drawn to some specific types of biologically active substances, methods for their isolation, description of physical-chemical and biochemical properties, their specific effect and physiological significance in relation to various tissues, organs and systems of the animal organism [21-23].

However, the problem of a systematic study of the metabolites of probiotic bacteria, due to its complexity, is still quite far from the final solution [24, 25].

The aim of the research is scientific and practical substantiation of molasses fermentation of by probiotic microorganisms to create a biologically active feed additive.

The research objectives included:

- study of the effectiveness of cultivation of Clostridium thermocellulociticus, Ruminococcus olbus, Clostridium lochheadii on a nutrient medium from beet molasses.

- obtaining a culture fluid with metabiotics of probiotic microorganisms.

- substantiation of the research results for the creation of biologically active additives for animals.

\section{METHODOLOGY}

The methodological basis of the research is the analysis of information and scientific assumptions on the cultivation of probiotic microorganisms in a high-carbohydrate nutrient medium with the determination of the main metabiotics during molasses fermentation. The studies were carried out in the laboratory "Livestock Biotechnology" of Kursk Federal Agrarian Scientific Center. The object of the research was sugar beet molasses $5 \%-10 \%-15 \%-20 \%-25 \%$. The probiotic microorganisms Clostridium thermocellulociticus, Ruminococcus olbus, Clostridium lochheadii, which are part of the enzymatic probiotic Celobacterin, were used for fermentation of molasses. The daily culture of microorganisms, which was standardized to $1 \times 10^{6} \mathrm{CFU} /$ $\mathrm{cm}^{3}$, was used to obtain a bacterial concentrate. The fermentation testing process was carried out according to the indicators of the mass fraction of fermentable sugars, determined by the calculation method (Industry Standard 18-395-82). The number of colonies forming units (CFU) was calculated in the Goryaev chamber according to the generally accepted method. The content of amino acids, vitamins and organic acids was determined by capillary electrophoresis on the device "Drops" manufactured by Lumex company according to the appropriate methods.

\section{RESULTS AND DISCUSSION}

Beet molasses has a complex and variable chemical composition, depending on the soil and climatic conditions of the growing season, fertilizers applied, harvesting methods, conditions and duration of storage of sugar beets, sugar technology and other factors. Molasses is a thick, viscous liquid of a dark brown color with a specific smell of caramel and melanoidins; beet molasses also has the smell of trimethylamine and other volatile amines formed during the decomposition of betaine. Of organic acids formic (0.1-1.2\%), acetic (0.6-1.3\%), propionic $(0.02-0.3 \%)$, butyric (up to $0.6 \%$ ), valerian (up to $0.2 \%$ ) and traces of about 20 aromatic acids are present. Acetic acid is formed in the process of defecation during alkaline decomposition of pectin substances and monosaccharides. However, most of the acetic acid, like other volatile acids and lactic acid, appears as a result of the vital activity of microorganisms. $50-60 \%$ of amino acids are transferred to molasses from beets. Aminobutyric acid is not found in beets and is formed from glutamic acid during decarboxylation when beets are processed. Glutamic acid easily cleaves water, turning into cyclic pyrrolidine carboxylic acid, in the form of which it is mainly $(75 \%)$ present in molasses. Molasses contains the following vitamins (average data, mg per $100 \mathrm{~g}$ ): biotin - 0.01, thiamine - 0.3, riboflavin - 0.04, pyridoxine -0.54 , nicotinic acid -5.1 , pantothenic acid 8.0, folic acid - 0.02. The average amount of mineral substances is $8.5 \%$, which are represented by macro- and microelements in various compounds. Molasses has a slightly alkaline or nearly neutral reaction $(\mathrm{pH} 8.9-7.2)$ and alkalinity of 2-0.5 mol / dm H $\mathrm{SO}_{4}$ in $1 \mathrm{~cm}^{3}$ [26].

The indicators of the chemical composition of sugar beets molasses, taken as the basis of the nutrient medium are shown in Table 1, due to the sufficient content of carbohydrate components for the nutrition of microorganisms.

Table 1. Indicators for testing the nutrient medium

\begin{tabular}{|c|c|c|}
\hline \multirow{2}{*}{ Indicators } & \multicolumn{2}{|c|}{ Test } \\
\cline { 2 - 3 } & benchmark & on the 8th day of cultivation \\
\hline Mass fraction of solids, $\%$ & 8.24 & 5.56 \\
\hline Hydrogen index, $\mathrm{pH}$ & 6.7 & 3.87 \\
\hline Mass fraction of the amount of fermented sugars, $\%$ & 53.7 & 33.7 \\
\hline Mass fraction of raw protein, $\mathrm{g} / \mathrm{l}$ & 5.35 & 5.7 \\
\hline
\end{tabular}

According to the results of testing the fermentation activity, the variability of the mass fraction of the amount of fermentable sugars in the range of $53.70 \%-33.79 \%$, the hydrogen index from 6.70 to 3.87 in the experimental samples of cultivated microorganisms was established, which indicates the activity of microorganisms in the nutrient medium. The studies have noted the variability of the dynamics of colony forming units (CFU 106) of probiotic microorganisms with respect to the nutrient medium, which was molasses in various concentrations (Fig. 1). 


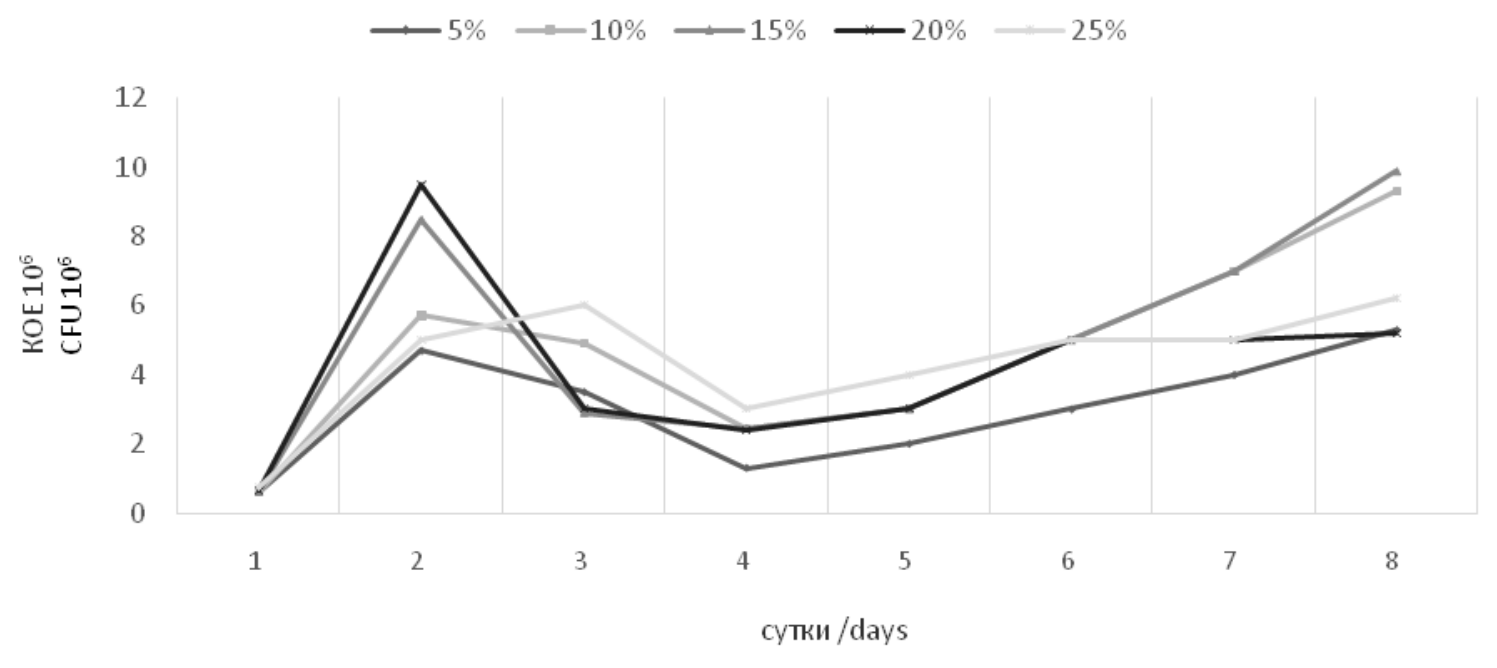

Figure 1. The variability of the dynamics of CFU of the "Cellobacterin" in relation to the concentration of molasses

When molasses is fermented by the complex of probiotic microorganisms "Cellobacterin", the maximum value of CFU 9,5x106 CFU / $\mathrm{cm}^{3}$ in $20 \%$ molasses is achieved on the 2nd - 3rd day, the minimum $5 \times 10^{6} \mathrm{CFU} / \mathrm{cm}^{3}$ in $25 \%$ molasses is associated with a specific type of microorganism and the specificity of its metabolism gives a sharp increase and then a decrease in microorganism numbers. A repeated increase in the number of CFU when using Cellobacterin was observed on the fourth day (1,3$\left.3 \times 10^{6} \mathrm{CFU} / \mathrm{cm}^{3}\right)$ and reached a maximum on the eighth day $\left(9,9 \times 10^{6} \mathrm{CFU} / \mathrm{cm}^{3}\right)$ with a molasses concentration of $15 \%$. In our opinion, the most optimal molasses concentration during fermentation with this probiotic is $15 \%$, since it provided the greatest number of CFU due to the fermentation of less available nutrients from 4 to 8 days. It was found that the number of probiotic microorganisms that make up the enzymatic probiotic Cellobacterin on the eighth day in $15 \%$ molasses-based nutrient medium is $9,9 \times 10^{6} \mathrm{CFU} / \mathrm{cm}^{3}$.

Analysis of the indicators given in table 2 marks the production of metabiotics in all cultivated microorganisms. It is known that amino acids are $16 \%$ composed of nitrogen, this is their main chemical difference from the other two most important nutrients carbohydrates and fats. Each protein in the body is unique and exists for special purposes. Proteins are not interchangeable. They are synthesized in the body from amino acids, which are formed as a result of the breakdown of proteins found in foods.

Table 2. Indicators of proteinogenic amino acids (mg / l)

\begin{tabular}{|c|c|c|}
\hline \multirow{2}{*}{ Indicators } & \multicolumn{2}{|c|}{ Test result } \\
\cline { 2 - 3 } & $\begin{array}{c}\text { Clostridium thermocellulociticus, } \\
\text { Ruminococcus olbus, Clostridium } \\
\text { lochheadii }\end{array}$ & $\begin{array}{c}\text { Molasses- } \\
\text { control }\end{array}$ \\
\hline Arginine & 51.80 & 18.30 \\
\hline Lysine & 6.30 & 0.62 \\
\hline Tyrosine & 25.40 & 0.44 \\
\hline Phenylalanine & 4.80 & 0.31 \\
\hline Histidine & 5.70 & 5.40 \\
\hline Leucine+Isoleucine & 57.90 & 14.90 \\
\hline Methionine & 14.90 & 2.10 \\
\hline Valine & 24.90 & 3.50 \\
\hline Proline & 54.20 & 2.40 \\
\hline Threonine & 115.80 & 1.50 \\
\hline Serine & 8.20 & 4.80 \\
\hline Alanine & 70.30 & 1.50 \\
\hline Glycine & 20.80 & 1.80 \\
\hline
\end{tabular}

Thus, amino acids rather than proteins are the most valuable nutrients, which is especially important when developing biologically active additives for the formation of microbiocenosis and nonspecific immunity in animals. In this case, the relationship of metabolism and the formation of immunity in general should be taken into account. Significant activity of the complex of microorganisms Clostridium thermocellulociticus, Ruminococcus olbus, Clostridium lochheadii has been established in the studies. Attention is drawn to the fact that a significant increase in the synthesis of amino acids by a complex of probiotic microorganisms included in the enzymatic probiotic Cellobacterin with respect to the control. It should be noted that $50-60 \%$ of amino acids are transferred to molasses from beets. The studies found a significant excess of Arginin 2.8 times, Tyrosine 57.7 times, Leucine + isoleucine 3.9 times, and other nonreplaceable and conditionally replaceable amino acids, which indicates a significant activity of probiotic microorganisms in the nutrient medium (Table 2). 
In this case, the significance of individual protein-forming acids for the animal organism and the resulting amino acid complex in protein metabolism should be taken into account. Indicators of organic acids produced by probiotic microorganisms have a high degree of difference with respect to the control (Fig. 2).

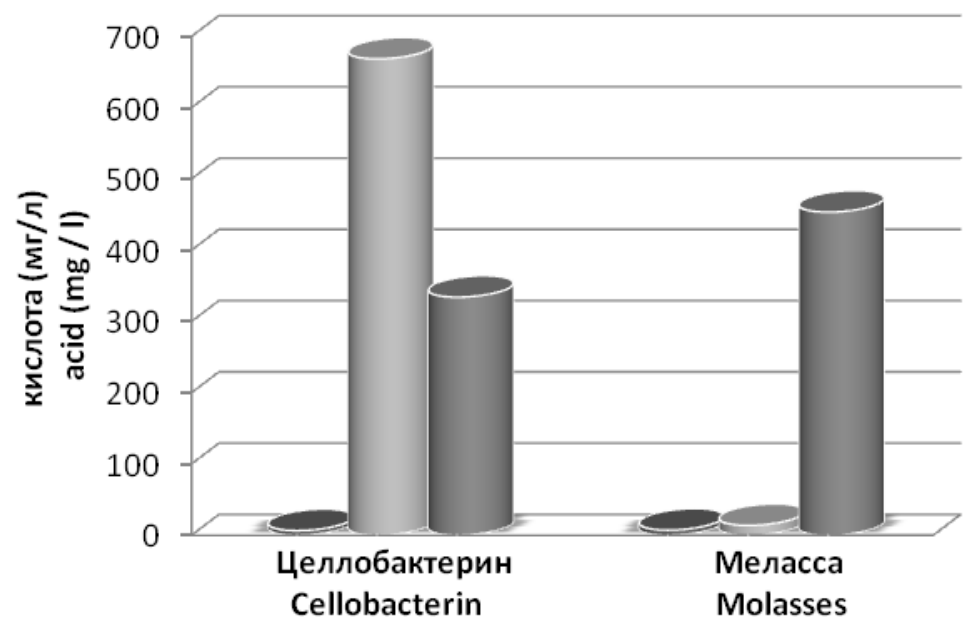

- щавелевая/ oxalic

янтарная / amber

- уксусная / acetic

Figure 2. Indicators of organic acids, mg/l

The qualitative composition of organic acids gives reason to predict their positive effect on the animal organism. At the same time, the content of oxalic acid in the test sample is slightly lower in relation to the control by $10.9 \%$. The content of acetic acid is lower than the control by $35.7 \%$. The amber acid value in the test sample relative to the control is increased by 48.8 times. The metabolites of the cultivated probiotic agents from the vitamin group are represented mainly by group "B". Their variability, both between the test samples and in relation to control should be noted (Fig. 3).

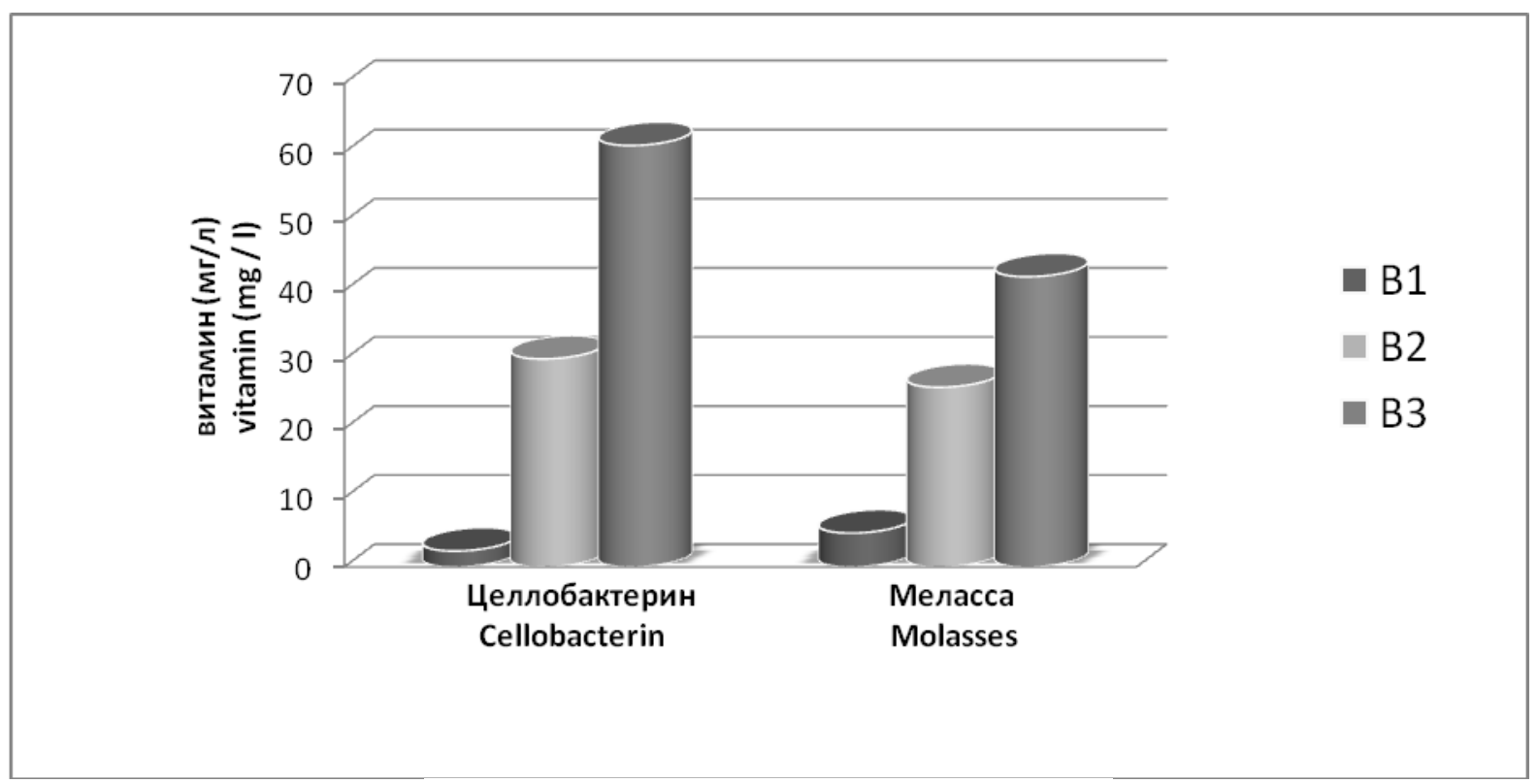

Figure 3. Indicators of water-soluble vitamins, mg / l

It should be noted that the culture fluid of the nutrient medium from molasses is adequate for the synthesis of amino acids, including essential, some vitamins and organic acids of the cultivated species of probiotic microorganisms, which is the basis for the creation of biologically active additives.

\section{CONCLUSION}

As a result of the studies, the possibility of obtaining metabiotics of probiotic microorganisms by cultivating them on a nutrient medium from beet molasses was established. The cultivation of probiotic microorganisms that are part of the enzymatic probiotic Cellobacterin is most effective in $15 \%$ molasses medium. According to the test results, the variability of the mass fraction of the 
amount of fermentable sugars in the range $53.70 \%$ $33.79 \%$, the hydrogen index from 6.70 to 3.87 in the experimental samples of the cultivated microorganisms was established, which indicates active metabolism of microorganisms in the nutrient medium. It should be noted that the nutrient medium from molasses has mainly carbohydrate components; therefore, the obtained metabiotic results are low. The qualitative composition of metabiotics provides the basis for further research in the development of biologically active additives for animals, including other nutrient media as well. The results of the research will contribute to the development of new approaches for the production of biologically active substances with subsequent use in animal husbandry, the creation of biologically active feed additives for the formation of microbiocenosis of the gastrointestinal tract in animals, including for the stimulation of nonspecific immunity.

\section{REFERENCES}

1. Ardatskaya MD., Stolyarova LG., Arkhipova EV., Filimonova OYu. Metabiotics as a natural development of the probiotic concept. Difficult patient 2017; 6-7, 15: 35-39.

2. Fisinin VI, Artemieva EA, Chebotarev II, Laptev GYu, Nikonov IN, Ilina LA, Mashentseva NG, Saninov AV, Klabukova DL, Yildirim EA, Novikova NI. Dietary probiotic Lactobacillus plantarum L-211 for farm animals. II. additive for piglets. Agricultural biology 2017; 52.2: 418-424.

3. Yoshimura $\mathrm{Yu}, \mathrm{Oda} \mathrm{M}$, Isobe $\mathrm{N}$. Influence of feed probiotics on the localization of cells containing Immunoreactive interleukin- 6 in the intestines of broiler chickens' text. Poultry TSM 2010; 47 (3): 250-255.

4. Andreeva AV, Nikolaeva ON. Probiotics, their influence on the intestinal microbiota. Scientific notes of the educational institution "Vitebsk order" Badge of Honor "State Academy of veterinary medicine" 2018; 54.1: 86-89.

5. Mituniewicz-Malek A, Zelinskaya D, Ziarno M. Probiotic monoculturel in fermented goat's milk drinks-sensory quality of the final product. International J. Dairy Technology 2019; 72.2: 240247.

6. Kesarkodi-Watson A, Kaspar H, Lategan MJ, Gibson L. Probiotics in aquaculture: necessity, principles and mechanisms of action and screening processes. Aquaculture 2008; 274.1: 1-14.

7. Bastionnoy K, Shleifer D. Probiotics work even in the presence of antibiotics. Mixed fodder 2020; 1: 109110.

8. Mikhalyuk A. Probiotics: purpose and prospects of use. Our agriculture 2019; 16 (216): 68-73.

9. Murlenkov NV. Russian and world market of feed probiotics. Scientific Journal of Young Scientists 2019; 2 (15): 5-8.

10. Yakovenko EP, Agafonova NA, Yakovenko AV, Ivanov AN, Soluyanova IP. Antibiotics, prebiotics, probiotics, metabiotics in excess bacterial growth in the small intestine. Difficult patient 2018; 16.4: 16-22.

11. Hixon M. Probiotics in the prevention of antibioticassociated diarrhea and Clostridium difficile infection. Therap. Adv. Gastroenterol. 2011; 4.3: 185197.
12. Melikidi VH, Tyurina DG, Selivanov DG, Novikova NI. Metabolites of probiotic bacteria are responsible for the effectiveness of the probiotic. Poultry Farming 2019; 09-10: 45-47.

13. Osintseva KR, Neverov OP. The role of probiotics in feeding farm animals. Youth and science 2019; 4: 39.

14. Gryazneva TN. Biologically active substances produced by bacteria of the genus Bacillus. Treating doctor 2013; 4: 54-63.

15. Efimova LV, Udalova TA. Effective microorganisms in feeding cattle and pigs. Krasnoyarsk: Krasnoyarsk research Institute of the Russian agricultural Academy; 2011.

16. Ushakova NA, Nekrasov RV, Pravdin VG, Kravtsova LZ, Bobrovskaya OI, Pavlov DS. A new generation of probiotic drugs for feed purposes. Fundamental research 2012; 1: 184-192.

17. Zhuk DS. Immune status and level of natural resistance in cattle when using "EM-VITA": Autoref...dis. kan. biol. nauk. Bryansk; 2018.

18. Karetkin BA, Doroshenko EO, Lanskikh AG, Tereshkova EA. Sorbed probiotics. Mechanism of action. Moscow: TD Delhi; 2020.

19. Marshall VM. Probiotics and prebiotics: scientific aspects. International journal of dairy technologies 2007; 60: 63-64.

20. Elijah Ohimine, Ruth Ofonko. Effect of probiotic and prebiotic feed additives on chickens. Health and intestinal microflora: review. International journal of animal husbandry and veterinary achievements 2012; 4 (2): 135-143.

21. Bondarenko VM, Chuprinina RP, Vorobieva MA. Mechanism of action of probiotic drugs. BIO drugs 2003; 3: 2-5.

22. Yildirim E, Ilina L, Novikova N, Tyurina D, Dubrovin A, Fillipova V, Laptev G. Norms of the content of microorganisms in the digestive tract of animals and birds. Mixed fodder 2019; 10: 70-74.

23. Schrezenmeir J, de Vrese M. Probiotics, prebiotics and synbiotics-approximation to the definition. Am. Jay Kline. Nutr. 2001; 73.l: 361-364.

24. Popov VS, Vorobyova NV. Correction of metabolism and microbiocenosis in pregnant sows. Veterinary medicine and feeding 2019; 4: 27-29.

25. Yates J. Naturalness and animal welfare. Animals 2018; 8.4: 53.

26. Paradeshi IS, Patil SN, Kohli SH, Chaudhary BL. The Influence of copper on the properties of probiotic bacteria Lactobacillus Helveticus CD6. International J. Dairy Technology 2018; 71.1: 204-212. 